\title{
Effects of G-Jitter Combined with Heat and Mass Transfer by Mixed Convection MHD Flow of a Second Grade Fluid in a Porous Medium
}

\author{
Faisal Salah1, 2, Z. A. Aziz", 3, K. K. Viswanathan²,3,* \\ Research, Universiti Teknologi Malaysia, 81310 UTM Johor Bahru, Johor, Malaysia. \\ Bahru, Johor, Malaysia. \\ * Corresponding author. Tel.:+60-163456966; email: visu20@yahoo.com \\ Manuscript submitted March 29, 2015; accepted October 15, 2015. \\ doi: 10.17706/ijapm.2015.5.4.234-242
}

${ }^{1}$ Department of Mathematics, Faculty of Science, University of Kordofan, Elobid, 51111, Sudan.

2 UTM Centre for Industrial and Applied Mathematics Ibnu Sina Institute for Scientific and Industrial

${ }^{3}$ Department of Mathematical Sciences, Faculty of Science, Universiti Teknologi Malaysia, 81310 UTM Johor

\begin{abstract}
The effects of g-jitter induced and combined with heat and mass transfer by mixed convection in microgravity situation on MHD flow second grade fluid in porous space is investigated for a particular system. This system consists of two heated vertical parallel infinite flat plates held at constant but different temperatures and concentrations. By using modified Darcy's law, the equations governing the flow are modelled. These equations are solved analytically for the induced velocity, temperature and concentration distributions. Similar result in the relevant literature relating to Newtonian fluid is also obtained as a special case of the present solution. Finally particular attention is given to the graphical results for the velocity profiles of the oscillating flow in the channel. The analysis on the variations of embedded flow parameters in the solution expressions are presented and discussed.
\end{abstract}

Key words: G-jitter, heat and mass transfer, mixed convection, second grade fluid; porous medium.

\section{Introduction}

The studies of non-Newtonian fluids have received considerable attention because of numerous applications in industry, geophysics and engineering. Some investigations are notably important in industries related to paper, food stuff, personal care products, textile coating and suspension solutions [1]-[3]. The non - Newtonian fluids have been mainly classified under the differential, rate and integrals types. The second grade fluids are the subclass of non - Newtonian fluids and are the simplest subclass of differential type fluids which can show the normal stress effects [4]-[7]. It was employed to study various problems due to their relatively simple structure. Moreover, one can reasonably hope to obtain exact solutions from this type of second grade fluid. This motivates us to choose the second grade model in this study. The exact solutions are important as these provide standard for checking the accuracies of many approximate solutions which can be numerical or empirical. They can also be used as tests for verifying numerical schemes that are developed for studying more complex flow problems [8]-[11].

Currently, we found great interest in the applications of the effects of complex body forces on fluid motion. Such forces exist, when a system with density gradients is subject to vibrations. The resulting buoyancy 
forces which are produced by the interaction of density gradients and the spatial and frequency distributions of the vibration-induced acceleration field on fluid motion is known as gravity modulation or g-jitter induced flow.

G-jitter is defined as the inertia effect due to quasi-steady, oscillatory or transient acceleration arising from aircraft's crew motions and machinery vibrations in parabolic aircrafts, space shuttles or other microgravity environments [12]-[15]. Sharidan et al. [16] has investigated the effects of g-jitter induced and combined with heat and mass transfer by mixed convection flow in microgravity situation for a simple system. This system consists of two heated vertical parallel infinite flat plates held at constant but different temperatures and concentrations for Newtonian fluid.

However, to the best for our knowledge, there are nocurrent attempts being made for viscoelastic fluids that take into account the phenomenon of g-jitter in free convection flows. These flows arise from the combined buoyancies due to thermal and chemical species concentration diffusion in a cavity.

The aim of this paper is to generate an exact analytical solution for the problem of laminar combined heat and mass transfer by mixed convection of a fully developed flow of a non Newtonian fluid. This flow is induced by a combination of g-jitter and an oscillating pressure gradient in a vertical channel of MHD flow second grade fluid. This fluid saturates the porous medium. We consider the g-jitter field to be constant in space, or else it would change with time harmonically. Finally the graphical results of the solution are plotted and discussed.

\section{Formulation of the Problem}

Let us consider a Cartesian coordinate system $(x, y)$ and the incompressible second grade fluid saturated porous half space bounded by an infinite plate at $y=0$ ( $y$-axis is taken normal to the plate). Moreover, the fluid is electrically conducting in the presence of an applied magnetic field $\boldsymbol{B}_{\circ}=\left(0,0, B_{\circ}\right)$. The magnetic Reynolds number is assumed small and hence the induced magnetic field is neglected. The flow is laminar mixed convection flow between two vertical parallel plates with uniform temperatures and concentrations at the walls with g-jitter fully developed.

The unsteady flow in a porous medium is governed by the following equations of continuity, motion, energy and concentration are:

$$
\begin{gathered}
\operatorname{div} \boldsymbol{V}=0, \text { (1) } \rho\left(\frac{\partial \boldsymbol{V}}{\partial t}+(\boldsymbol{V} . \nabla) \boldsymbol{V}\right)=-\nabla p+\operatorname{div} \boldsymbol{S}+\boldsymbol{J} \times \boldsymbol{B}+\boldsymbol{R}+\left[\beta_{T}\left(T-T_{0}\right)+\beta_{C}\left(C-C_{0}\right)\right] g^{*}(t) \\
\rho c_{p} \frac{\partial T}{\partial t}=k \frac{\partial^{2} T}{\partial y^{2}}, \frac{\partial C}{\partial t}=D \frac{\partial^{2} C}{\partial y^{2}}
\end{gathered}
$$

In the above equations $\boldsymbol{V}=(u, 0,0)$ is the velocity filed, $t$ is time, $\rho$ is the fluid density, $p$ is the pressure, $\boldsymbol{S}$ is the extra stress tensor, $\boldsymbol{J}$ is the current density, $\boldsymbol{B}=B_{\circ}+b$ is the total magnetic field, $B$ 。 and $b$ are the applied and induced magnetic fields respectively and $\boldsymbol{R}$ is the Darcy resistance, $g^{*}(t)$ is the gravity acceleration is given by $g^{*}(t)=g_{0} \sin (\omega t)$ where $g_{0}$ is the magnitude,$\omega$ is the frequency of the g-jitter field, $\beta_{T}$ and $\beta_{C}$ are coefficients of thermal and mass expansions respectively, $T$ is the temperature, $c_{p}$ is the specific heat capacity, $k$ is the thermal conductivity, $C$ is the 
concentration and $D$ is the mass diffusivity. It is assumed that at time $t=0$ the flow is at the constant temperature $T_{0}$ and constant concentration $C_{0}$.

For the second grade fluid we have the extra stress tensor $\boldsymbol{S}$ given in [5] and [10] as

$$
S=\mu \mathbf{A}_{1}+\alpha_{1} A_{2}+\alpha_{2} A_{1}^{2}
$$

where $\mu$ is the dynamic viscosity and $\alpha_{i}(i=1,2)$ are material constants satisfying $\alpha_{1} \geq 0, \alpha_{1}+\alpha_{2}=0$, $\boldsymbol{A}_{1}$ and $\boldsymbol{A}_{2}$ are the first two Rivlin - Eriksen tensors and the velocity field $\boldsymbol{V}$ is assumed in the form

$$
\boldsymbol{V}=(u(y, t), 0,0)
$$

\section{Exact Solution of the Problem}

Introducing the dimensionless variables

$$
\zeta=\frac{y}{h}, f=\frac{u}{u_{0}}, \xi=\frac{x}{h}, \tau=\frac{v}{h^{2}} t, P=\frac{p}{\rho v u_{0} / h}, \theta=\frac{T-T_{0}}{T_{2}-T_{0}}, \phi=\frac{C-C_{0}}{C_{2}-C_{0}}, \Omega=\frac{\omega h^{2}}{v}, g(\tau)=\frac{g(t)}{g_{0}}
$$

Our problem becomes

$$
\begin{gathered}
\beta \frac{\partial f}{\partial \tau}=-\frac{\partial P}{\partial \xi}+\frac{\partial^{2} f}{\partial \zeta^{2}}+\alpha \frac{\partial^{3} f}{\partial \tau \partial \zeta^{2}}-\left(M^{2}+\frac{1}{B}\right) f+\frac{G r}{\operatorname{Re}} g(\tau)(\theta+N \phi), \\
\operatorname{Pr} \frac{\partial \theta}{\partial \tau}=\frac{\partial^{2} \theta}{\partial \zeta^{2}}, \quad S c \frac{\partial \phi}{\partial \tau}=\frac{\partial^{2} \phi}{\partial \zeta^{2}}
\end{gathered}
$$

where $\beta=1+\frac{\alpha}{B}, \alpha=\frac{\alpha_{1}}{\rho h^{2}}, \frac{1}{B}=\frac{h^{2} \varphi}{K}, M=\frac{\sigma B_{0}^{2} h^{2}}{\rho v}, G r=\frac{g_{0} \beta_{T}\left(T-T_{0}\right) h^{3}}{v^{2}}, \quad \operatorname{Re}=\frac{u_{0} h}{v}$ $N=\frac{\beta_{C}\left(C-C_{0}\right)}{\beta_{T}\left(T_{2}-T_{0}\right)}, \operatorname{Pr}$ and $S c$ are the Prandtl and Schmidt numbers, respectively.

Equations (6) and (7) are subject to the following boundary conditions:

$$
f=0, \theta=r_{T}, \phi=r_{C} \text { on } \zeta=0, \quad f=0, \theta=1, \phi=1 \text { on } \quad \zeta=1, r_{C}=\frac{C_{1}-C_{0}}{C_{2}-C_{0}}, r_{T}=\frac{T_{1}-T_{0}}{T_{2}-T_{0}}
$$

The solution of equation (6) and (7) are derived as follows:

$$
f(\zeta, \tau)=\frac{e^{i \Omega \tau}}{m^{2}(1+i \alpha \Omega)}\left[\begin{array}{c}
\left(\frac{\partial F}{\partial \xi}-\frac{G r}{\operatorname{Re}}(1+N)\right) \frac{\sinh (m \zeta)}{\sinh (m)}+\left(\frac{\partial F}{\partial \xi}-\frac{G r}{\operatorname{Re}}\left(r_{T}+N r_{C}\right)\right) \frac{\sinh (m(1-\zeta))}{\sinh (m)} \\
+\frac{G r}{\operatorname{Re}}\left(1-r_{T}+N\left(1-r_{C}\right)\right) \zeta-\frac{\partial F}{\partial \xi}+\frac{G r}{\operatorname{Re}}\left(r_{T}+N r_{C}\right)
\end{array}\right]
$$


where $m=\sqrt{\frac{i \Omega \beta+M+\frac{1}{B}}{1+i \alpha \Omega}}$,

$\frac{\partial F}{\partial \xi}=\frac{m^{3}(1+i \alpha \Omega) \sinh (m)}{2 \cosh (m)-m \sinh (m)-2}+\frac{G r}{\operatorname{Re}} \frac{\left(1+r_{T}+\left(1+r_{C}\right) N\right)}{2}$ if we used the condition of conservation of mass $\int_{0}^{1} \Phi(\zeta) d \zeta=1$

The expressions (8) for MHD viscous fluid $(\alpha=0), \beta=1$ in a porous space are

$$
f(\zeta, \tau)=\frac{e^{i \Omega \tau}}{\eta^{2}}\left[\begin{array}{c}
\left(\frac{\partial F}{\partial \xi}-\frac{G r}{\operatorname{Re}}(1+N)\right) \frac{\sinh (\eta \zeta)}{\sinh (\eta)}+\left(\frac{\partial F}{\partial \xi}-\frac{G r}{\operatorname{Re}}\left(r_{T}+N r_{C}\right)\right) \frac{\sinh (\eta(1-\zeta))}{\sinh (\eta)} \\
+\frac{G r}{\operatorname{Re}}\left(1-r_{T}+N\left(1-r_{C}\right)\right) \zeta-\frac{\partial F}{\partial \xi}+\frac{G r}{\operatorname{Re}}\left(r_{T}+N r_{C}\right)
\end{array}\right],
$$

with

$$
\eta=\sqrt{i \Omega+M+\frac{1}{B}}, \text { and } \frac{\partial F}{\partial \xi}=\frac{\eta^{3} \sinh (\eta)}{2 \cosh (\eta)-\eta \sinh (\eta)-2}+\frac{G r}{\operatorname{Re}} \frac{\left(1+r_{T}+\left(1+r_{C}\right) N\right)}{2} .
$$

If we put $M=\frac{1}{B}=\mathrm{O}$ into Eq. (10) we recover the exact solution obtained by Sharidan et al. ([16] Eq. (11)) as given by

$$
f(\zeta, \tau)=\frac{e^{i \Omega \tau}}{\delta^{2}}\left[\begin{array}{c}
\left(\frac{\partial F_{2}}{\partial \xi}-\frac{G r}{\operatorname{Re}}(1+N)\right) \frac{\sinh (\delta \zeta)}{\sinh (\delta)}+\left(\frac{\partial F_{2}}{\partial \xi}-\frac{G r}{\operatorname{Re}}\left(r_{T}+N r_{C}\right)\right) \frac{\sinh (\delta(1-\zeta))}{\sinh (\delta)} \\
+\frac{G r}{\operatorname{Re}}\left(1-r_{T}+N\left(1-r_{C}\right)\right) \zeta-\frac{\partial F_{2}}{\partial \xi}+\frac{G r}{\operatorname{Re}}\left(r_{T}+N r_{C}\right)
\end{array}\right]
$$

with

$$
\delta=\sqrt{i \Omega} \text {, and } \frac{\partial F_{2}}{\partial \xi}=\frac{\delta^{3} \sinh (\delta)}{2 \cosh (\delta)-\delta \sinh (\delta)-2}+\frac{G r}{\operatorname{Re}} \frac{\left(1+r_{T}+\left(1+r_{C}\right) N\right)}{2} .
$$

In the case of steady flow $\Omega=0$, the gravity field is constant which corresponds to the buoyancy driven flow under terrestrial conditions. The solution of this particular case is contained in Eq. (8) as follows:

$$
f(\zeta)=\frac{1}{m_{1}^{2}}\left[\begin{array}{c}
\left(\frac{\partial F_{1}}{\partial \xi}-\frac{G r}{\operatorname{Re}}(1+N)\right) \frac{\sinh \left(m_{1} \zeta\right)}{\sinh \left(m_{1}\right)}+\left(\frac{\partial F_{1}}{\partial \xi}-\frac{G r}{\operatorname{Re}}\left(r_{T}+N r_{C}\right)\right) \frac{\sinh \left(m_{1}(1-\zeta)\right)}{\sinh \left(m_{1}\right)} \\
+\frac{G r}{\operatorname{Re}}\left(1-r_{T}+N\left(1-r_{C}\right)\right) \zeta-\frac{\partial F_{1}}{\partial \xi}+\frac{G r}{\operatorname{Re}}\left(r_{T}+N r_{C}\right)
\end{array}\right],
$$


where

$$
m_{1}=\sqrt{M+\frac{1}{B}}, \frac{\partial F_{1}}{\partial \xi}=\frac{m_{1}^{3} \sinh \left(m_{1}\right)}{2 \cosh \left(m_{1}\right)-m_{1} \sinh \left(m_{1}\right)-2}+\frac{G r}{\operatorname{Re}} \frac{\left(1+r_{T}+\left(1+r_{C}\right) N\right)}{2}
$$

But for the steady state for hydrodynamic second grade in nonporous case, (i.e. $M=\frac{1}{B}=0$ ), the solution takes the form

$$
f(\zeta)=6\left(\zeta-\zeta^{2}\right)+\frac{G r}{\operatorname{Re}}\left[\left(1-r_{T}+N\left(1-r_{C}\right)\right)\left(\frac{\zeta}{12}-\frac{\zeta^{2}}{4}+\frac{\zeta^{3}}{6}\right)\right]
$$

The above solution (12) can be shown to be equivalent to the steady solution of the Newtonian fluid. See shardian et al. [16], eq. (12).

\section{Results and discussion}

This section concerns with the variations of embedded flow parameters in the solution expressions. Hence Fig. 1 to Fig. 9 have been displayed in order to illustrate such variations.

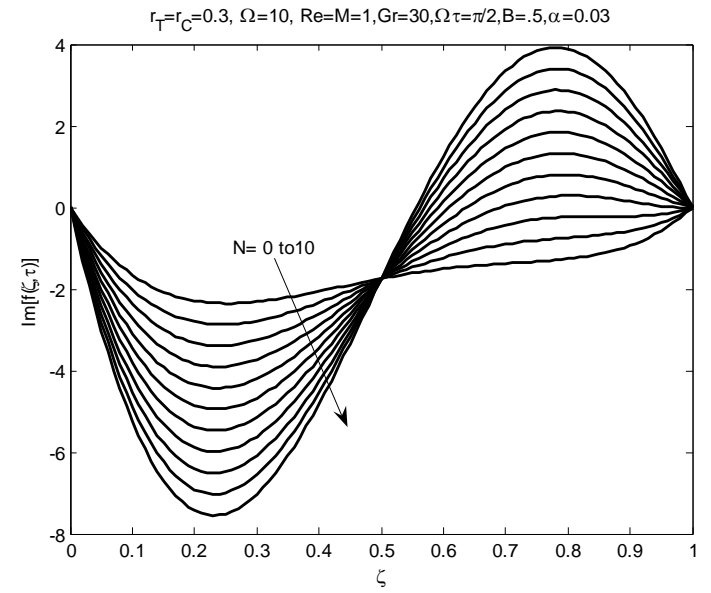

Fig. 1. Effects of $N>0$ (aiding buoyancies).

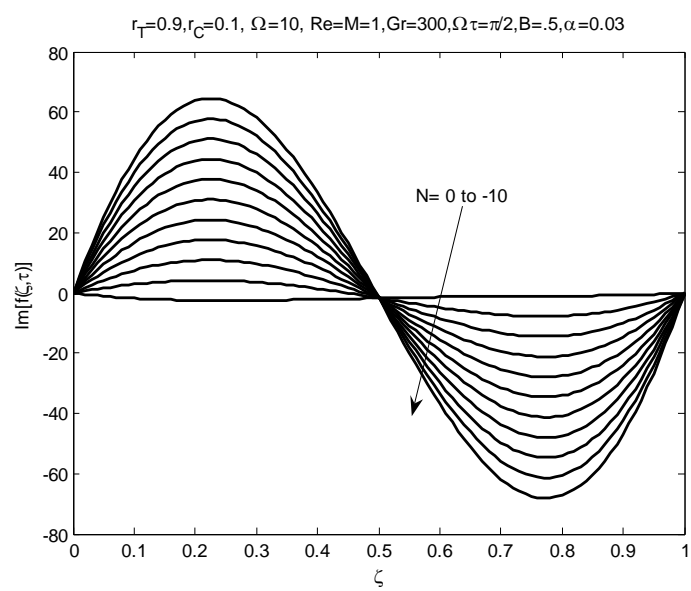

Fig. 3. Effects of $N<0$ and $r_{C}<r_{T}$.

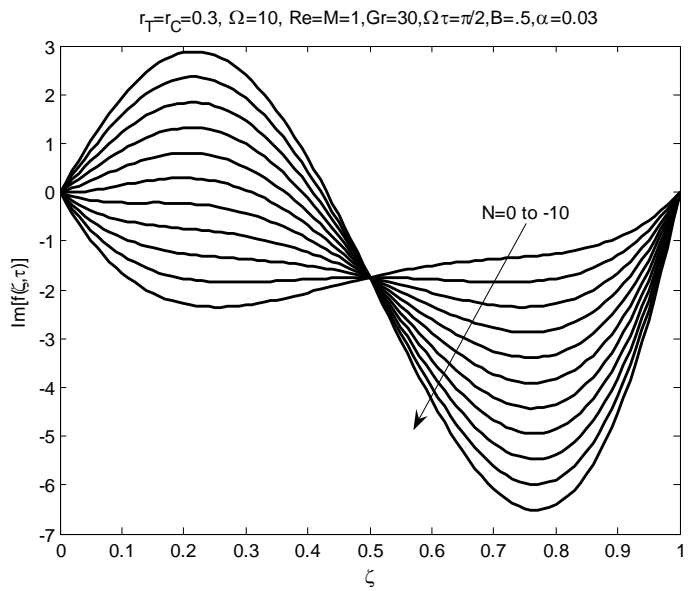

Fig. 2. Effects of $N<0$ (opposing buoyancies).

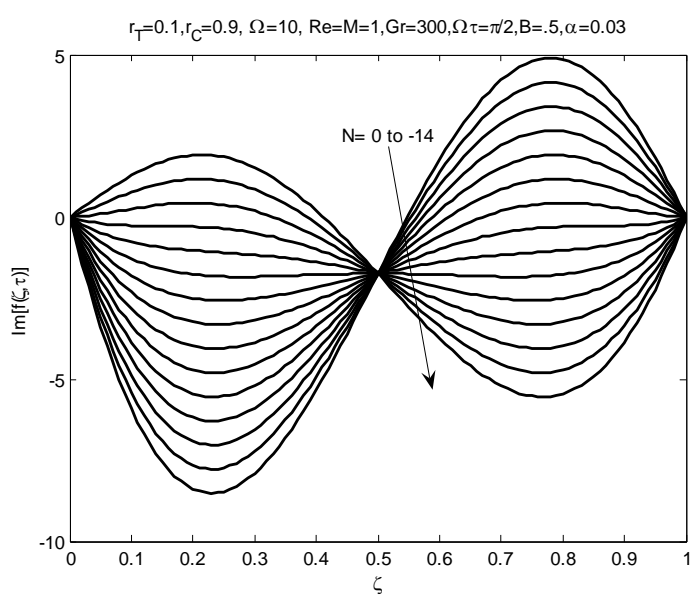

Fig. 4. Effects of $N<0$ and $r_{T}<r_{C}$. 
Fig. 1 and 2 shown that for, $r_{C}=r_{T}$ the flow is reversed close to the left wall for $N>0$ (aiding buoyancies) and close to the right wall for opposing buoyancies case when $N<0$, respectively. However, when $N<0$ and $r_{C}>r_{T}$, the flow is reversed only near the right wall, while for $N<0$ and $r_{C}<r_{T}$, the flow is reversed close to the both walls as can be seen from Fig. 3 and Fig. 4.

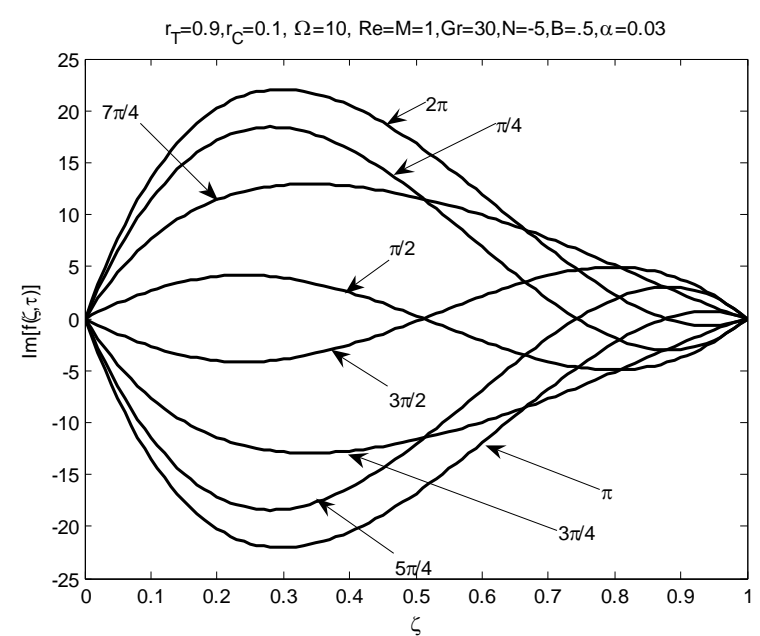

Fig. 5. Effects of $\Omega \tau$ when $r_{C}<r_{T}$.

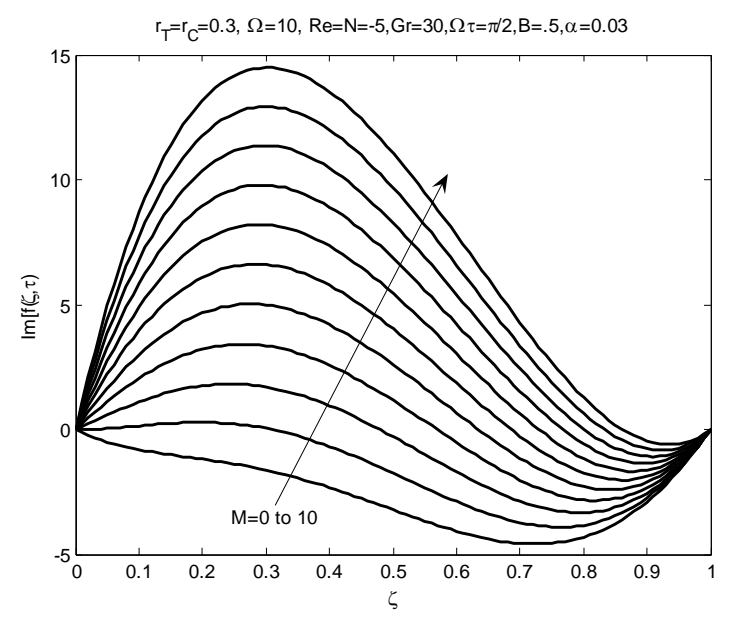

Fig. 7. Effects of $M$ when $r_{T}=r_{C}$.

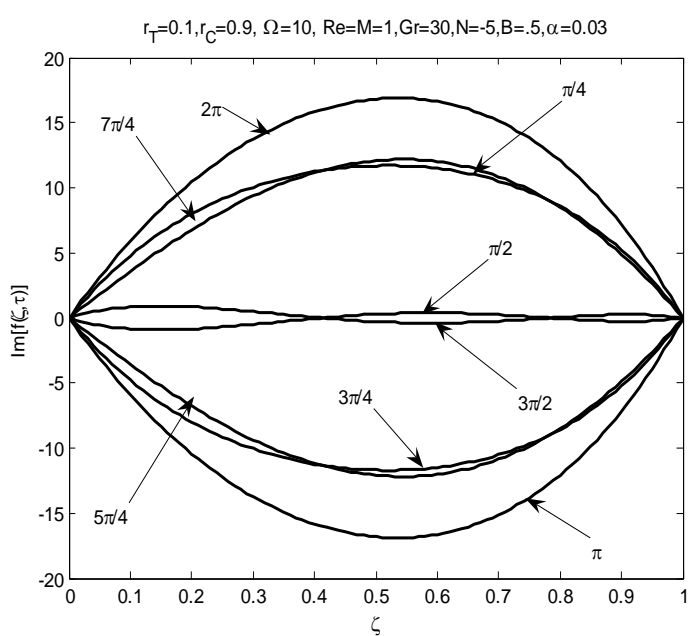

Fig. 6. Effects of $\Omega \tau$ when $r_{T}<r_{C}$.

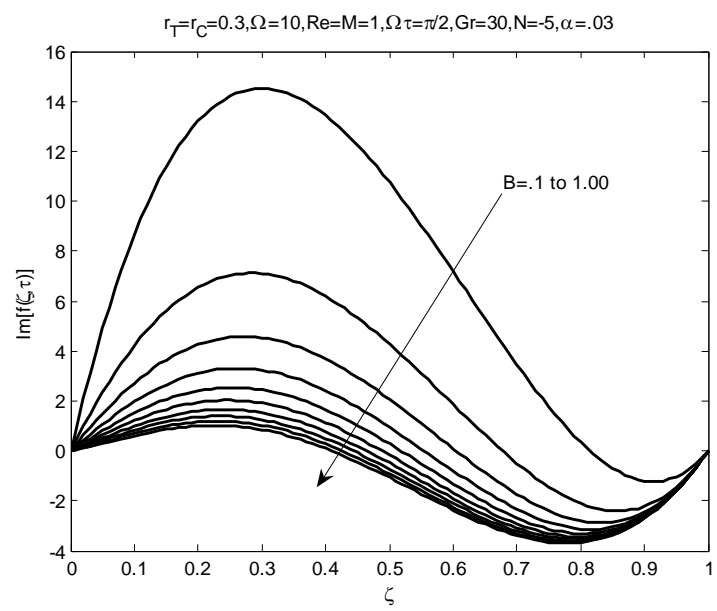

Fig. 8. Effects of $B$ when $r_{T}=r_{C}$.

Very interesting situations can be seen in Fig. 5 and Fig. 6 for $N<0$ namely $N=-5$, say, and different values of $\Omega \tau$ varying from $\Omega \tau=\pi / 4$ to $\Omega \tau=2 \pi$. We can see here that there is only a small reversed flow close to the right wall for $r_{C}<r_{T}$ and $G r / \operatorname{Re}=30$ (Fig. 5) but it increases when $\mathrm{Gr} / \mathrm{Re}=300$ (Fig. 6). On the other hand, for $r_{C}>r_{T}$ there is full reversed flow for some values of $\Omega \tau$ (Fig. 5) and partial reversed flow (Fig. 6) when $\mathrm{Gr} / \mathrm{Re}$ increased.

Fig. 7 is prepared to see the effects of applied magnetic field (Hartman number) $M$ on the velocity profile. Keeping the other parameters are fixed and varying $M$, it is noted that the velocity profile decreases by increasing the magnetic field parameter $M$. Clearly, we observe that with increasing values of $M$, the velocity profile of $f(\zeta)$ decreases, in fact this is because of the effects of transverse magnetic 
field on an electrically conducting fluid which gives rise to a resistive type force called the Lorentz force which tends to slow down the motion of the fluid.

Fig. 8 indicates the variations of the porous parameter $B$. it is noted that by increasing the porous parameter $B$, this would lead to decrease in the velocity profile.

Fig. 9 shows the effects of parameter $\alpha$ of velocity profile when the other parameters are fixed. It is interesting to notice that by increasing in the parameter $\alpha$, this would lead to the increase in the velocity profile. This is because of the fact that increasing values of $\alpha$ would reduce the friction forces, and thus assists the flow of the fluid considerably; and hence the fluid moves with greater velocity.

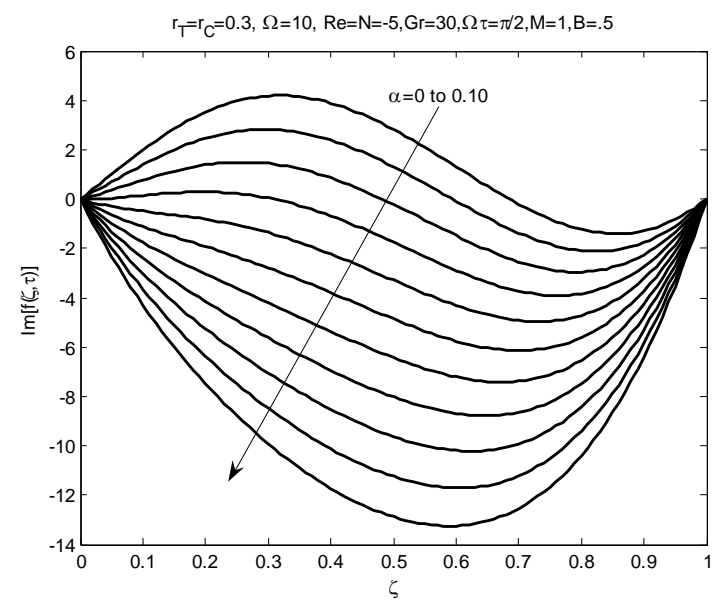

Fig. 9. Effects of $\alpha$ when $r_{T}=r_{C}$.

\section{Concluding Remarks}

In this article, the effect of g-jitter induced combined heat and mass transfer by mixed convection MHD second grade flow in microgravity for a simple system consisting of two heated vertical parallel infinite flat plates held at constant but different temperatures and concentrations has been studied. In addition the graphical results are plotted and discussed. From the presented analysis, the main observations are described as below:

1) The effect of $B$ and $\alpha$ on the velocity profile components leads to an opposite effect to $M$.

2) The results corresponding to viscous fluid can be obtained by choosing $\alpha=0$.

3) The steady state solution for hydrodynamic second grade fluid it shown to be equivalent to the steady state case of viscous fluid.

\section{Acknowledgements}

The authors thankfully acknowledge the financial support from MOHE, GUP Research Grant Vote No. Q.J130000.2509.05H78 under Research Management Centre (RMC), Universiti Teknologi Malaysia for completion of this research work. Dr Faisal is thankful to University of Kordofan and Sudanese government for financial support.

\section{References}

[1] Zhao, S. Y., \& Jian, Z. L. (1998). Numerical research on the coherent structure in the viscoelastic second-order mixing layers. Applied Mathematics and Mechanics, 19(8), 717-723.

[2] Hussain, M., Hayat, T., Asghar. S., \& Fetecau, C. (2010). Oscillatory flows of second grade fluid in a 
porous space. Nonlinear Analysis: Real World Applications, 11(4), 2403-2414.

[3] Khan, M., Naheed, E., Fetecau, C., \& Hayat, T. (2008). Exact solutions of starting flows for second grade fluid in porous medium. International Journal of Nonlinear Mechanics, 43(9), 868-879.

[4] Salah, F., Aziz, Z. A., \& Dennis, L. (2011). New exact solutions for MHD Transient Rotating flow of a Second grade fluid in a porous medium. Journal of Applied Mathematics.

[5] Tan, W. C., \& Masuoka, T. (2005). Stokes' first problem for a second grade fluid in a porous half space with heated boundary. International Journal of Nonlinear Mechanics, 40(4), 515-522.

[6] Salah, F., Aziz, Z. A., \& Dennis, L. (2011). New exact solution for Rayleigh — Stokes problem of Maxwell fluid in a porous medium and rotating frame. Results in Physics, 1(1), 9-12.

[7] Rajagopal, K. R., \& Gupta, A. S. (1981). On a class of exact solutions to the equations of motion of a second grade fluid. International Journal of Engineering Science, 19(7), 1009-1014.

[8] Erdogan, M. E., \& Imrak, C. E. (2005). On unsteady unidirectional flows of a second grade fluid. International Journal of Nonlinear Mechanics, 40(10), 1238-1251.

[9] Fetecau, C., Hayat, T., Fetecau, C., \& Ali, N. (2008). Unsteady flow of a second grade fluid between two side walls perpendicular to a plate. Nonlinear Analysis: Real World Applications, 9(3), 1236-1252.

[10] Salah, F., Aziz, Z. A., \& Dennis, L. (2011). Accelerated flow of magneto hydrodynamic (MHD) second grade fluid over an oscillating plate in a porous medium and rotating frame. International Journal of the Physical Sciences, 6(36), 8027-8035.

[11] Fetecau, C., \& Fetecau, C. (2005). Starting solutions for unsteady unidirectional flows of a second grade fluid. International Journal of Engineering Science, 43(10), 781-789.

[12] Fetecau, C., Sharat, C. P., \& Rajagopal, K. R. (2007). A note on the flow induced by a constantly accelerating plate in an Oldroyd-B fluid. Applied Mathematical Modelling, 31(4), 647-654.

[13] Sharidan, S., Amin, N., \& Pop, I. (2007). G-Jitter free convection flow in the stagnation-point region of a three-dimensional body. Mechanics Research Communications, 34(2), 115-122.

[14] Amin, N. (1988). The effect of g-jitter on heat transfer. Proceedings of the Royal Society of London: Vol. 1856. A. Mathematical and Physical Sciences (pp. 151-172).

[15] Chamkha, A. J. (2003). Effects of heat generation on g-jitter induced natural convection flow in a channel with isothermal or isoflux walls. Heat and Mass Transfer, 39(7), 553-560.

[16] Sharidan, S., Amin, N., \& Pop, I. (2005). G-jitter fully developed combined heat and mass transfer by mixed convection flow in a vertical channel. International Communications in Heat and Mass Transfer, 32(5), 657-665.

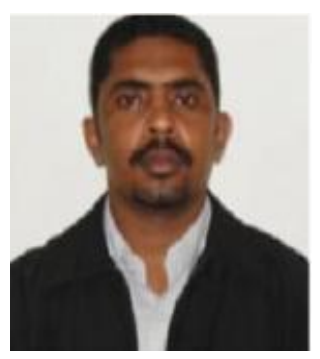

Faisal Salah was born in 1977 in Dongola, Sudan. He received his B.Sc. in mathematics from University of Aljazeera, Wad-Madani, Sudan, Sept. 2001 and the M.Sc. from Elneelien University, Khartoum, Sudan, and the Ph.D from Universiti Teknologi Malaysia (UTM), Johor, Malaysia. From 2013-2015, he did his post doctoral research at UTM under Project title: Homotopy analysis method on non linear equation of physical significance. His research areas are in fluid mechanics and mathematical physics. $\mathrm{He}$ published many research articles in International Journals.

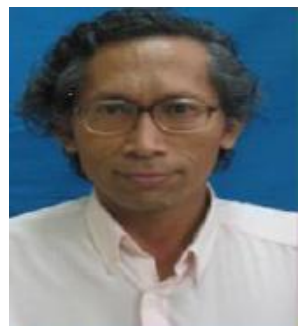

Zainal Abdul Aziz received his B.Sc. in mathematics from University of Manchester Institute of Science and Technology, Manchester, England and the M.Sc. and Ph.D from Universiti Kebangsaan Malaysia, Bangi, (UKM), Malaysia. Currently he is a director of UTM Centre for Industrial and Applied Mathematics (UTM-CIAM) at Universiti Teknologi Malaysia, Johor Bahru, Malaysia. He published many research articles in 
reputed International Journals. His areas of interest includes applied analysis \& mathematical physics (AAMP), functional integrals in mathematical physics, formalization of feynman integral, applications in theoretical physics: basic string theory, non linear waves: mathematics and physics of solitons: extension of IST (inverse scattering technique), multidimensional solitons, integrable systems (infinite dimensional hamiltonian systems), strings, hirota \& sato formalisms, mathematical modelling of surface waves, ocean \& seismic waves, engineering \& industrial mathematics and philosophy of mathematical sciences \& applicable mathematics education.

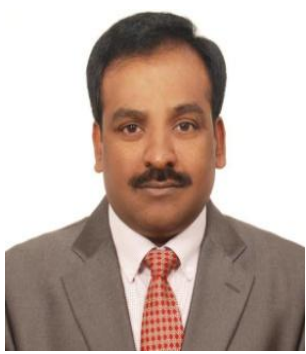

K. K. Viswanathan was born in 1962 in Vellore District, India. He received his B.Sc. in mathematics from University of Madras and the M.Sc. and Ph.D from Anna University, India. Later he was a project associate in Indian Institute of Science, Bangalore. He served as a lecturer in Crescent Engg. College and as asst. professor in SRM University, India. He did his post doctoral research and worked as a foreign professor for three years at Inha University, South Korea . At present he serves as associate professor in the UTM Centre for Industrial and Applied Mathematics, Department of mathematical Sciences at Universiti Teknologi Malaysia, Johor Bahru, Malaysia. He published many research articles in reputed international journals. His areas of interest includes vibration of plates, shells and the application of numerical techniques in Engineering problems. 